\title{
Demographic characteristics of patients with hand-foot-and- mouth disease. Atypical cases series
}

\author{
Asuman Gürkan, M.D. ${ }^{a}$, Mehtap Acar, M.D. ${ }^{b}$ and Saliha Şenel, M.D. ${ }^{c}$
}

\begin{abstract}
Hand-foot-and-mouth disease (HFMD) is a common childhood exanthem. Various types of lesions and widespread distribution in atypical cases have been described, but data on the predilection of lesion localizations in atypical cases are insufficient. We aimed to describe the demographic features of patients with HFMD, and to characterize lesion localizations in patients with atypical eruptions treated at an outpatient dermatology clinic of a pediatric hospital, between November 2011 and August 2013.The study included 67 patients. Mean age of the patients was 34 months and there was a male predominance $(60 \%)$. All the patients had eruptions on hands, feet, and mouth. Children aged $<24$ months had involvement of the diaper area and extremities, which was significantly higher than those aged 24-48 months and $>48$ months $(\mathrm{P}<0.0001$ and $\mathrm{P}=0.011$, respectively). None of the patients had serious systemic complications.

Key words: hand, foot and mouth disease, atypical, diapers rash.
\end{abstract}

http:/ /dx.doi.org/10.5546/aap.2015.eng.e211

\section{INTRODUCTION}

Hand-foot-and-mouth disease (HFMD) is an acute viral illness characterized by fever, intraoral vesicles/erosions, and papulovesicles on the palms of the hands and soles of the feet. ${ }^{\text {SSince }}$ first report in 1957 by Robinson et al., it has become a well-known childhood disease. ${ }^{2}$ Human enterovirus 71 and coxsackie virus (CV)-A16 are the most common pathogens that cause HFMD. ${ }^{3}$ HFMD is typically mild and self-limited but sometimes serious complications occur. ${ }^{4-5}$ HFMD primarily affects children aged $<5$ years. $^{4}$

a. Department of Dermatology.

b. Department of Pediatrics.

Dr. Sami Ulus Maternity and Children's Health and Diseases Training and Research Hospital.

c. Associated Proffesor, Department of Pediatrics, Yildirim Beyazit University School of Medicine.

Ankara, Turkey.

E-mail Address:

Asuman Gürkan, M.D.: asucolak@yahoo.com

Funding: None.

Conflict of interest: None.

Received: 12-13-2014

Accepted: 3-5-2015
In children with infectious diseases cutaneous lesions usually provide clues for early diagnosis, ${ }^{6,7}$ which largely relies on clinical manifestations and is beneficial for pediatric patients, as it limits the necessity for invasive examinations. ${ }^{6}$

As reports of atypical morphologies and distribution of eruptions related to enteroviral infections are increasing, the present study aimed to evaluate the demographic features of patients with HFMD and the predilection of lesion localizations in patients with atypical eruptions.

\section{MATERIALS AND METHODS}

This descriptive study included patients aged 0-16 years that were diagnosed as HFMD and evaluated at Dr. Sami Ulus Maternity and Children Hospital, Departments of Dermatology and Pediatrics, Ankara, Turkey, between November 2011 and August 2013. Patients that met the clinical criteria for typical and atypical HFMD were included. Typical disease was defined as oral and/or perioral erosions and vesicles, and exanthem on the palms of the hands and soles of the feet, whereas atypical disease was defined as exanthem on distinct sites other than $\mathrm{oral} /$ perioral region, palms of the hands and soles of the feet with typical disease. Atypical cases were diagnosed based on initial experience and awareness of atypical cases in Turkey during May to August 2012. Patients were excluded if their clinical presentation could be explained (clinically and serologically) by another specific illness, such as varicella, herpes simplex virus, herpes zoster, contact dermatitis, atopic dermatitis, bullous impetigo, or other bullous diseases.

Electronic patient records were accessed to obtain demographic, epidemiologic, and clinical data. The distribution of sesions was assessed, including lesion type, location, and extension. Localization of disease was categorized as oral/perioral, hands-feet, diaper area (genitalinguinal area and buttocks), trunk, extremities, and scalp. Patients were classified into 3 age groups ( $<24$ months, $24-48$ months, and $>48$ months). Statistical analysis was performed using SPSS v.11.5 for Windows, (SPSS, Inc., Chicago, IL, 
USA). Pearson's chi-square test or Fisher's exact test was used to determine differences between categorical variables. All $\mathrm{P}$ values are 2 -sided associations and the level of statistical significance was set at $\mathrm{P}<0.05$. The study protocol was approved by the Institutional Review Board.

\section{RESULTS}

The study included 67 patients with a mean age of $34 \pm 27$ months (range: 7-132 months). Forty patients $(60 \%)$ were male. All the patients had involvement of the typical sites. Patient demographic data and distribution of the lesions are shown in Table 1. Among the patients, 46 (69\%) were diagnosed between May to August 2012. Of these 46 patients, 29 had atypical disease. Among the 15 patients diagnosed after August 2012, ${ }^{9}$ had atypical disease. Among the atypical cases,

TABLE 1. Demographic features of the patients and distribution of lesions

\begin{tabular}{lc}
\hline Variables & Patients (n= 67) \\
Mean age (months) & $34.0 \pm 26.7$ \\
Age range (months) & $7-132$ \\
Age groups & \\
$\quad<24$ months & $30(44.8 \%)$ \\
$24-48$ months & $24(35.8 \%)$ \\
$\quad>48$ ay months & $13(19.4 \%)$ \\
Gender & \\
$\quad$ Male & $40(59.7 \%)$ \\
$\quad$ Female & $27(40.3 \%)$ \\
Lesion localization & \\
Oral/perioral & $67(100.0 \%)$ \\
$\quad$ Hands-feet & $67(100.0 \%)$ \\
Diaper area & $37(55.2 \%)$ \\
Extremities & $29(43.3 \%)$ \\
Trunk & $16(23.9 \%)$ \\
Scalp & $2(3.0 \%)$ \\
\hline
\end{tabular}

diaper area involvement was significantly more common in the female patients $(\mathrm{P}=0.002)$. There wasn't a significant difference in involvement of hands-feet, trunk, or scalp according to gender $(\mathrm{P}=0.245, \mathrm{P}=0.794$, and $\mathrm{P}=0.512$, respectively). The frequency of diaper area involvement was significantly higher in the patients aged $<24$ months $(\mathrm{P}<0.001)$ than in those aged $24-48$ months and $>48$ months $(\mathrm{P}=0.004$ and $\mathrm{P}<0.001$, respectively) (Figure 1), whereas there wasn't a significant difference in the frequency of diaper area involvement between the patients aged 2448 months and $>48$ months $(\mathrm{P}=0.305)$.

Extremity involvement was significantly more common in the patients aged $<24$ months $(\mathrm{P}=0.011)$, as compared to those aged 24-48 months and $>48$ months $(\mathrm{P}=0.013$ and $\mathrm{P}=0.015$, respectively) (Figure 2) . There wasn't a significant
FIgURE 1. Papulovesicles on diaper area

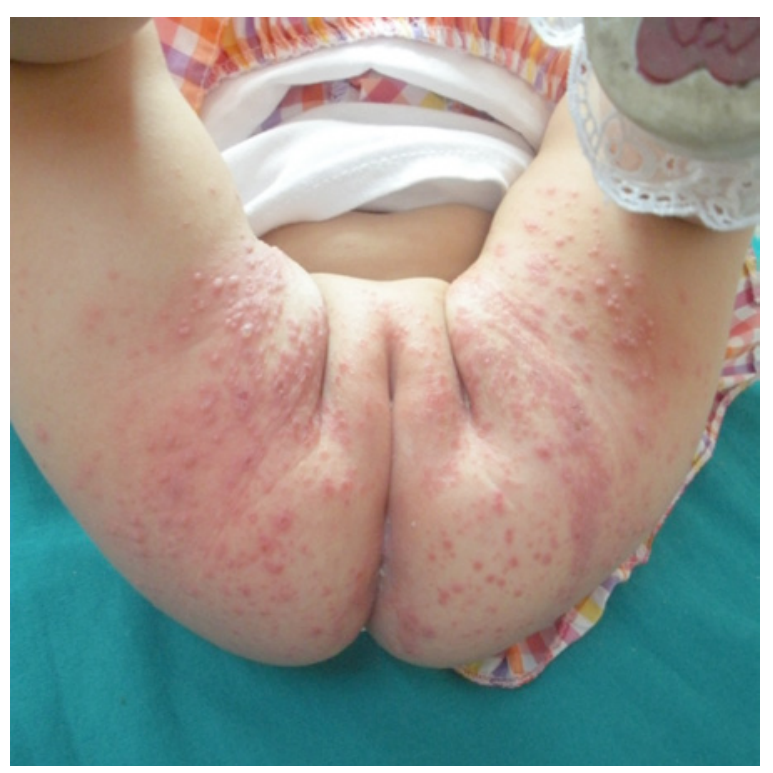

TABLE 2. Lesion localization on atypical regions according to age groups

\begin{tabular}{|c|c|c|c|c|}
\hline Localization & $\begin{array}{c}<24 \text { months } \\
(n=30)\end{array}$ & $\begin{array}{l}24-48 \text { months } \\
\quad(n=24)\end{array}$ & $\begin{array}{l}>48 \text { months } \\
\quad(n=13)\end{array}$ & $\mathbf{P}$ \\
\hline Diaper area & $24(80.0 \%)^{\mathrm{a}, \mathrm{b}}$ & $10(41.7 \%)^{\mathrm{a}}$ & $3(23.1 \%)^{b}$ & $<0.001^{*}$ \\
\hline Extremities & $19(63.3 \%)^{a, b}$ & $7(29.2 \%)^{a}$ & $3(23.1 \%)^{b}$ & $0.011^{*}$ \\
\hline Trunk & 11 (36.7\%) & $4(16.7 \%)$ & $1(7.7 \%)$ & $0.072^{*}$ \\
\hline Scalp & $1(3.3 \%)$ & - & $1(7.7 \%)$ & $0.339^{* *}$ \\
\hline
\end{tabular}

*Pearson's chi-square test. **Likelihood ratio test.

a Significant difference between $<24$ months and $24-48$ months $(\mathrm{P}<0.05)$.

b Significant difference between $<24$ months and $>48$ months $(\mathrm{P}<0.05)$. 
difference in extremity involvement between the patients aged $24-48$ months and $>48$ months $(\mathrm{P}=1,000)$.

There weren't any significant differences in involvement of the trunk or scalp according to age groups $(\mathrm{P}=0.072$ and $\mathrm{P}=0.339$, respectively). The localization of lesions according to age groups is shown in Table 2. Only two patients had scalp involvement; one was aged 13 months and had widespread disease, and the other was aged 132 months (11 years) and aside from hands-feet and mouth involvement, the scalp was the only atypical localization. Only one patient was hospitalized because of widespread disease. None of the patients had serious systemic complications.

\section{DISCUSSION}

HFMD is a viral illness characterized by intraoral vesicles or erosions, and papulovesicles on the palms of the hands and soles of the feet. ${ }^{1,5} \mathrm{It}$ is an acute, highly contagious disease that usually occurs in summer and fall. ${ }^{5}$ Cutaneous lesions vary from erythematous papules to oval vesicles. Exanthem of the mouth is present in almost all patients. The disease resolves within one week without any treatment. ${ }^{1}$

HFMD commonly affects children aged $<5$ years. ${ }^{5}$ A large-scale study from China reported that children aged $<5$ years constituted the largest group of patients, with a peak incidence at age 1 year, and that the approximately $65 \%$ of the patients were male. ${ }^{4}$ In another study $89.75 \%$ of HFMD patients were aged $<4$ years. ${ }^{8}$ Other studies confirmed the younger age $(<5$ years $)$ and

FIGURE 2. Papulovesicles on extremity

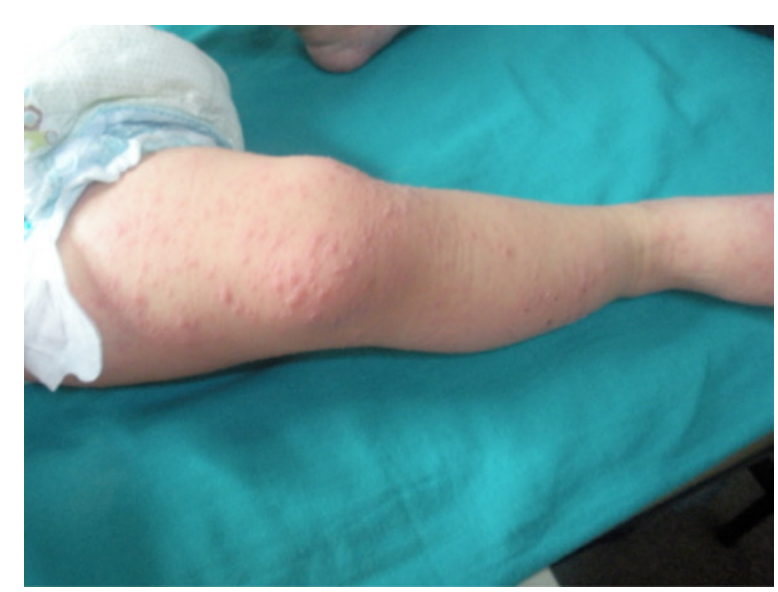

male predominance either. ${ }^{9,10}$ Similar findings were observed in the present study. Younger age $(<5$ years) predominance was related to the differences in serum antibodies according to age groups. ${ }^{4}$ Additionally, a seroprevalence study from Germany reported that $66 \%$ of children aged 1-4 years did not posses neutralizing antibodies to $\mathrm{CV}-\mathrm{A} 16 .{ }^{11}$

Since 2004, there has been an increase in the number of reports of a more severe form of HFMD associated with CV-A6. ${ }^{5}$ Widespread and severe disease commonly occurs in outbreaks, which usually occur in late fall or winter ${ }^{1}$; yet, there was a marked increase in the number of patients with HFMD in the summer of 2012 in Turkey ${ }^{12}$ and in the present study $69 \%$ of cases were diagnosed in summer. A striking finding is the increased number of atypical patients ( 9 of 15) diagnosed after this period. Along with the expectation of the occurrence atypical HFMD cases with different strains in the future, we think it is critically important to identify atypical cases.

Based on our experience, perioral erosions and/or vesicles are striking presentations. Diaper area rash appeared before exanthem on the palms of the hands and soles of the feet. Lesions other than papulovesicles can be predominant in patients with HFMD, as Mathes et al. reported. ${ }^{13}$

The diaper area was the most commonly affected site in the present study's patients aged $<24$ months. Buttocks may also be involved in typical disease ${ }^{5}$ but in atypical cases the lesions may extent to all diaper area. Accentuation of viral exanthems in inflamed regions, especially in cases of blood-borne viruses, has been reported. Lesions of HFMD have a predilection for localizing in regions of inflammation. ${ }^{14}$ Kar et al. described the demographic features of patients and the clinical presentations of HFMD due to CV-A16 during an outbreak in Bhubaneswar, India in 2009. ${ }^{3}$ They reported the buttocks was the most common and severely affected region, followed by the knees. Buttocks/groin/perineum was involved in $76 \%$ of Mathes et al.'s patients. ${ }^{13}$ Another clinical study on severe HFMD with systemic complications reported that the hips were affected in $53 \%$ of patients. ${ }^{15}$ The buttocks were affected in $31 \%$ of patients with atypical HFMD in a hospital-based prospective study. ${ }^{6}$

Moreover, there wasn't a significant difference in the frequency of trunk and scalp involvement according to age group, and the scalp was the least commonly affected. The extremities were the most commonly affected region $(100 \%)$ in atypical 
HFMD cases due to CV-A6 during an outbreak between 2011 and 2012 in North America. ${ }^{13}$ In the present study the frequency of extremity involvement in the patients aged $<24$ months was also high $(43.3 \%)$, and lesions with variable morphology, such as papules, purpura, and vesicles, were observed on the extremities area.

Physicians must be aware of and able to recognize atypical presentation of HFMD in patients. ${ }^{5}$ HFMD disease must be included in the differential diagnosis of acute onset of vesicles or erosions in the diaper area in infants with perioral/oral lesions.

\section{REFERENCES}

1. Lott JP, Liu K, Landry ML, Nix WA, et al. Atypical handfoot-and-mouth disease associated with coxsackievirus A6 infection. J Am Acad Dermatol 2013;69(5):736-41.

2. Robinson CR, Doane FW, Rhodes AJ. Report of an outbreak of febrile illness with pharyngeal lesions and exanthem: Toronto, summer 1957; isolation of group A Coxsackie virus. Can Med Assoc J 1958;79(8):615-21.

3. Kar BR, Dwibedi B, Kar SK. An outbreak of hand, foot and mouth disease in Bhubaneswar, Odisha. Indian Pediatr 2013;50(1):139-42.

4. Deng T, Huang Y, Yu S, Gu J, et al. Spatial-temporal clusters and risk factors of hand, foot, and mouth disease at the district level in Guangdong Province, China. PLoS One 2013;8(2):e56943.

5. Stewart CL, Chu EY, Introcaso CE, Schaffer A, et al.
Coxsackievirus A6-induced hand-foot-mouth disease. JAMA Dermatol 2013;149(12):1419-21.

6. Huang WC, Huang LM, Lu CY, Cheng AL, et al. Atypical hand-foot-mouth disease in children: a hospital-based prospective cohort study. Virol J 2013;10:209.

7. OoiMH, Wong SC, Lewthwaite P, Cardosa MJ, etal.Clinical features, diagnosis, and management of enterovirus 71 . Lancet Neurol 2010;9(11):1097-105.

8. Qiaoyun F, Xiongfei J, Lihuan L, Angao X. Epidemiology and etiological characteristics of hand, foot and mouth disease in Huizhou City between 2008 and 2011. Arch Virol 2013;158(4):895-9.

9. Chen SC, Chang HL, Yan TR, Cheng YT, et al. An eightyear study of epidemiologic features of enterovirus 71 infection in Taiwan. Am J Trop Med Hyg 2007;77(1):188-91.

10. Zou XN, Zhang XZ, Wang B, Qiu YT. Etiologic and epidemiologic analysis of hand, foot, and mouth disease in Guangzhou city: a review of 4,753 cases. Braz J Infect Dis 2012;16(5):457-65.

11. Rabenau HF, Richter M, Doerr HW. Hand, foot and mouth disease: seroprevalence of Coxsackie A16 and Enterovirus 71 in Germany. Med Microbiol Immunol 2010;199(1):45-51.

12. PolatEkinci A, ErbudakE, BaykalC. [Asignificant increase in the frequency of hand-foot-mouth disease in stanbul in June 2012]. Turkderm 2013;47(4):192-3.

13. Mathes EF, Oza V, Frieden IJ, Cordoro KM, et al. "Eczema coxsackium" and unusual cutaneous findings in an enterovirus outbreak. Pediatrics 2013;132(1):e149-57.

14. Messner J, Miller JJ, James WD, Honig PJ. Accentuated viral exanthems in areas of inflammation. J Am Acad Dermatol 1999;40(2 Pt 2):345-6.

15. Tian H, Yang QZ, Liang J, Dong SY, et al. Clinical features and management outcomes of severe hand, foot and mouth disease. Med Princ Pract 2012;21(4):355-9. 